\title{
Management of patients with acute pharyngitis
}

\author{
Bernardo Ejzenberg *
}

In the present issue of Jornal de Pediatria, Santos \& Berezin ${ }^{1}$ discuss one of the most relevant themes related to general pediatrics: the management of patients with acute pharyngitis. The authors assessed the accuracy of a rapid test and of clinical parameters in the diagnosis of Streptococcus pyogenes pharyngitis, comparing it with the throat swab culture (gold standard). The conclusions concur with those of other studies, which demonstrate better accuracy of the rapid test. ${ }^{2-4}$ There is a direct implication on pediatric management, since the diagnosis of streptococcal pharyngitis determines the administration of antibiotics for the prevention of late complications - rheumatic disease and acute glomerulonephritis. ${ }^{4}$ Next, some considerations will be made about the etiologic diagnosis and antibiotic therapy used in patients with acute pharyngitis.

\section{Use of lab tests for detection of Streptococcus pyogenes infection}

The laboratory etiologic identification of Streptococcus pyogenes has been employed on a large scale in the USA for more than one decade. ${ }^{3,4}$ It is recommended by the scientific committees for cardiology, infectology and pediatrics. ${ }^{5-7}$ The rapid tests, which detect bacterial antigens, are predominantly used; have advantages over the throat swab culture, are less time-consuming and easily applied (in minutes), in addition to having a low cost. The accuracy of rapid tests has gradually improved, as shown in the article at issue; today, false-positive results are not common, and false-negative ones are quite few. ${ }^{4}$ The nondetection of some group $\mathrm{C}$ and $\mathrm{G}$ beta-hemolytic streptococci, which are also rheumatogenic, has limited importance, due to the

\footnotetext{
* Professor, Children's Institute, Hospital das Clínicas, School of Medicine, Universidade de São Paulo (USP), São Paulo, SP. Coordinator of Research and Publications of the Division of Pediatrics of Hospital Universitário, Universidade de São Paulo (USP), São Paulo, SP, Brazil.

Suggested citation: Ejzenberg B. Management of patients with acute pharyngitis. J Pediatr (Rio J). 2005;81:1-2.
}

infrequency of these infections. Another restrictive factor is the detection of Streptococcus pyogenes carriers, which does not seem to occur in the rapid tests, as described by Santos \& Berezin. ${ }^{1}$

There is some uncertainty about the indication of the rapid test for the diagnosis of pharyngitis: should it be indicated for all cases or only for those with suspected streptococcal infection $?^{4}$

The extent of the etiological investigation depends on the goals to be achieved with the available technical and financial resources. It should be noted that $20 \%$ of children with streptococcal pharyngitis ( $5 \%$ of the total number of cases of acute pharyngitis) do not show remarkable symptoms, but can develop late complications. ${ }^{1-4}$ Actually, some of the cases of rheumatic disease do not have a previous history of severe pharyngitis. ${ }^{5}$

In populational terms, the tests have a unit cost under $\mathrm{R} \$ 5.00$ (around U\$ 1.80), which is largely compensated for by the reduced use of antibiotics, as discussed further ahead. A decrease in the use of antimicrobials reduces the antibiotic resistance of Streptococcus pneumoniae and Haemophilus influenzae and of other bacteria found in the skin and in the digestive tract. The lower occurrence of adverse effects produced by unnecessary medications should also be considered as an advantage of the rapid test. Put all together, these facts lead to the recommendation of the rapid test to all patients with acute pharyngitis, regardless of its clinical intensity.

\section{Evaluation of pharyngitis by the pediatrician based only on clinical signs and symptoms}

This is a usual type of evaluation, but it results in increased uncertainty about the occurrence of streptococcal pharyngitis - in approximately $50 \%$ of cases. ${ }^{4}$ This was also observed in a study conducted at Santa Casa de Misericórdia de São Paulo, where pediatricians prescribed $55 \%$ of antibiotics for a group of children with a $24.4 \%$ infection rate. Additionally, pediatricians did not recognize $5 \%$ of streptococcal infections, which were regarded as viral infections, a characteristic that was also observed in other 
studies. ${ }^{1-4}$ Thus, based only on clinical symptomatology, $1 / 3$ of all cases of pharyngitis are inappropriately treated with antibiotics and, in general, $40 \%$ of the diagnoses and antibiotic therapies are incorrect. The use of standardized clinical scores, such as the CENTOR score, helps minimize some of these errors. ${ }^{4}$

\section{Other aspects related to the diagnosis and management of children with acute pharyngitis}

Usually the patients brought to a pediatrician are those presenting with a fever, as described by the authors. ${ }^{1}$ Fever was observed in $93 \%$ of cases, and in each one of them, it indicated the nature of the disease but not its focus (or foci). The physical examination, which determines the focus in the pharynx, may not fully detect the extent of the infection in the body. In their turn, microbiological studies of the pharynx also do not assess the remainder of the airways and of the body. Such limitations should be taken into consideration.

Some children with pharyngitis have rhinopharyngosinusitis or develop it in the short run, which is poorly detected on physical examination. ${ }^{8}$ This may have special importance in case of patients who remain feverish during the course of pharyngitis and who do not have Streptococcus pyogenes in their pharynx. In some cases, plain $\mathrm{x}$-rays of the paranasal cavities are employed to characterize rhinopharyngosinusitis, but to almost no avail.9,10 The symptomatology is often viral at the beginning, but after three days of fever, approximately $50 \%$ of the patients present with a secondary infection caused by Streptococcus pneumoniae, Haemophilus influenzae or Moraxella catarrhalis. ${ }^{8}$ Therefore, the old practice of prescribing antibiotics for use after three days of febrile pharyngitis is understandable. For cases of pharyngitis that become afebrile during the course of the disease, the simultaneous occurrence of sinus infection has limited importance; since at least 10 days should be allowed before management is started. ${ }^{8}$

Bacteremia is another clinical problem to be considered in a patient with pharyngitis. This often occurs among infants, who were not included in the article at issue. Bacteremia affects 1 to $3 \%$ of infants and has a lower incidence in older children, even if pharyngitis is of viral etiology. The rapid test does not detect this clinical situation.

For these reasons, the rapid test is believed to define the etiology of pharyngitis and improve the indication of antibiotic therapy, but it does not put an end to the uncertainties about the administration of antibiotics. In fact, there exists a patient with pharyngitis to be treated and not only a pharyngitis. U.S. studies show that after a throat swab culture with a negative result only $50 \%$ of physicians recommend the discontinuation of the antibiotic in use. ${ }^{1}$ This might be seen as an error of conduct, but also as a precaution, due to the suspicion of non-streptococcal bacterial infection of the adjacent airways or bacteremia. Clinical signs suggestive of these situations include progressive or persistently high-grade fever, shivering, deterioration of the general health status, and increase of nasal discharge and congestion. ${ }^{8}$
In conclusion, the study carried out by Santos \& Berezin ${ }^{1}$ corroborates the results obtained by other authors and shows that the treatment of children with acute pharyngitis has been improved. For initial examination, the rapid test or throat culture, if possible, should be used. Clinical evaluation should investigate the patient as a whole, upper and lower airways, and the signs of systemic dissemination of the infection. The decision for antibiotic therapy is based on these aspects. The course of the disease is dynamic, may have complications, and the family should be informed about it. The follow-up of febrile cases is mandatory, and clinical reassessments are necessary.

Currently, in the city of São Paulo, at least five pediatric emergency departments perform the rapid test. Because of that, some mothers have urged pediatricians to request the test before a possible prescription of antibiotics. The reasons why this test is not widely used in our setting are trivial: antibiotics are prescribed on a large scale. Also, the cost of antibiotics is incurred by the family or by the government, whereas the test is paid by the service where the patient is treated.

The advantage of the rapid diagnostic test to public health is quite clear, as it reduces the use of antibiotics and decreases the speed for the development of resistance to several bacteria. Based on the evidence presented, the recommendation to perform screening tests for Streptococcus pyogenes in cases of acute pharyngitis, before the prescription of antibiotic therapy, is just a matter of time.

\section{References}

1. Santos AG, Berezin I. Comparação entre métodos clínicos e laboratoriais no diagnóstico das faringotonsilites estreptocócicas. J Pediatr (Rio J). 2005;81:23-8.

2. Gerber MA, Shulman ST. Rapid diagnostic of pharingitis caused by group A streptococci. Clin Microbiol Rev. 2004;17:571-80.

3. Shulman ST. Acute streptococcal pharyngitis in pediatric medicine: current issues in diagnosis and management. Paediatr Drugs. 2003; Suppl 1:13-23.

4. Merril B, Kalsberg G, Jankovski TA, Danis T. What is the most effective diagnostic evaluation of streptococcal pharyngitis? J Fam Pract. 2004;53:737-8.

5. Dajani A, Taubert K, Ferrieri P. Committee on Rheumatic Fever, Endocarditis, and Kawasaki Disease of the American Heart Association. Treatment of acute streptococcal pharyngitis and prevention of rheumatic fever: a statement for health professionals. Pediatrics. 1995;96:758-64.

6. Bisno AL, Gerber MA, Gwaltney JM, Kaplan EL, Schwartz RH. Infectious Diseases Society of America. Practice guidelines for the diagnosis and management of group A streptococcal pharyngitis. Clin Infect Dis. 2002;35:113-25.

7. American Academy of Pediatrics. Group A Streptococcal Infections. In: Pickering LK, ed. Red Book: 2003 Report of the Committee on infectious diseases. 26th ed. Elk Grove Village (IL): American Academy of Pediatrics; 2003. p. 573-84.

8. Ejzenberg B, Sih T, Haetinger, RG. Conduta diagnóstica e terapêutica na sinusite da criança. J Pediatr (Rio J). 1999;75: 419-32.

9. Ejzenberg B. Epidemia de sinusites. Pediatria (São Paulo). 1998;20:69.

10. Haetinger RG, Krakauer AM, Sih T, Lotufo JP, Ejzenberg B, Okay Y. Avaliação radiológica de sinusite em crianças: indicações e limitações. Rev Med Hosp Univ. 1999;9:7-13. 